\title{
La memoria en tiempos de la complejidad
}

\author{
Oscar Ochoa Flores
}

\section{Resumen}

Ésta es una breve reflexión sobre la memoria colectiva a partir de algunos principios básicos de la complejidad y la transdisciplina, por ejemplo, la recursividad, la dialogicidad y los niveles de realidad, expuestos en términos tan sencillos como el tema lo permite. El primer apartado desarrolla los principios en términos de un pensamiento abierto a otras formas de conocer y se centra en varios niveles de la realidad, frente a un pensamiento lineal y mecanicista como el heredado del siglo XIX con respecto al tema de la memoria.

El segundo apartado se enfoca en describir la memoria como resultado de proceso evolutivo a partir de una estrategia de cooperación que facilitó a los seres vivos sobrevivir, lo que se expresa de tal forma en el trabajo grupal de neuronas, personas y especies.

En el tercero y último apartado se observa la parte emocional de la memoria, necesaria para fijar un recuerdo en el contexto social y cómo puede movilizar palabras o acciones para darle sentido a las sociedades cuando éstas se hallan en situaciones de extremas en las cuales es necesario recurrir a la memoria para reafirmar el origen y defender su presente.

Palabras clave: memoria, complejidad, transdisciplina, vida, sociedad.

\section{MEMORY IN TIMES OF COMPLEXITY}

\begin{abstract}
This is a brief reflection on the collective memory based on some basic principles of complexity and transdisciplinarity, for example recursion, dialogicity and levels of reality, exposed in terms as simple as the subject allows. The first section develops the principles in terms of a thought open to other ways of knowing and focuses on several levels of reality, as opposed to a linear and mechanistic thought as inherited from the nineteenth century with respect to the subject of memory.

The second section focuses on describing memory as the result of an evolutionary process based on a cooperation strategy that facilitated living beings to survive, which is expressed in such a way in the group work of neurons, people and species.

In the third and last section we observe the emotional part of the memory, necessary to fix a memory in the social context and how it can mobilize words or actions to give meaning to societies when they are in extreme situations in which it is necessary recourse to memory to reaffirm the origin and defend its present.
\end{abstract}

Key words: memory, complexity, transdiscipline, life, society.

DOI: http://doi.org/10.22201/codeic.16076079e.2018.v19n2.a6 


\section{Oscar Ochoa Flores}

foscar@hotmail.com

Licenciado en Diseño de la Comunicación Gráfica por la Universidad Autónoma Metropolitana (UAM) Xochimilco (1999), licenciado en Etnología por la Escuela Nacional de Antropología e Historia (ENAH) (2009), maestro en Comunicación y Política por la UAM Xochimilco. Doctorante en Antropología Social por la ENAH. Artículos publicados: "Las dimensiones de la intervención: acontecimiento, alteridad y diálogo" (2013), "La construcción del poder desde el teatro callejero" (2010), "Los hombres de papel" (2006). Distinciones obtenidas: Medalla al Mérito Universitario, UAM Xochimilco (2014) y Beneficiario del Programa de Estímulo a Creadores del IMCINE (2007), entre otros.

\section{Ideas complejas para la actualidad}

Todavía son escasos los esfuerzos por generar conocimiento más allá de la ciencia desarrollada en occidente, mediante la búsqueda del diálogo con otras formas de sabiduría como la que nos han legado los pueblos ancestrales; y todavía son más raros aquellos que lo hacen desde la complejidad, que en palabras de su principal exponente la define como "la incertidumbre en el seno de los sistemas ricamente organizados. Tiene que ver con los sistemas semi-aleatorios cuyo orden es inseparable de los azares que incluyen. La complejidad está así ligada a una cierta mezcla de orden y desorden..." (Morin, 2001: 35) cuyos principios rebasan el pensamiento lineal con el cual hemos sido educados toda la vida. La complejidad nos llevaría a pensar en la comunicación permanente entre los distintos niveles de la realidad,

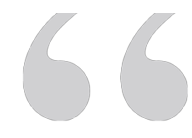

\section{La cooperación} entre las células cerebrales para evocar recuerdos requiere de conexiones al azar, en lo que se conoce como plasticidad cerebral a considerar la relación recíproca entre causas y efectos, opuestos y complementarios, etcétera; pero debemos aceptar también que esto resulta muy difícil para muchos e incluso imposible para otros.

Hablar desde la complejidad y la transdisciplina significa, en sí, un riesgo que no muchos deciden correr, pues de un proyecto que involucre estas características se pueden obtener cuatro o cinco proyectos más. Debemos reconocer que, si ya es complicado lidiar con proyectos que dependen de una sola disciplina o de varias disciplinas o de la convergencia entre éstas, un proyecto transdisciplinario nos obliga a salir de los límites del conocimiento propio y pensar de forma distinta para buscar aquellas ideas que nos permitan elaborar conceptos o formas de investigar adecuadas, tal es el caso de un tema tan complejo como el que nos ocupa: la memoria colectiva.

En términos generales, la memoria se define como "la capacidad que tienen los organismos de mantener una respuesta a un estímulo sensorial des- 
podemos hablar y conocer; así como la memoria lingüística afecta en la genética y el conocimiento a partir de la evolución que las lenguas han tenido a lo largo de la humanidad, modificando las zonas especializadas para el habla en el cerebro y la laringe. La memoria cognitiva ha influido en la memoria genética y la memoria lingüística a partir del conocimiento que la humanidad ha desarrollado sobre la genética y el lenguaje, lo que ha hecho posible revertir ciertas enfermedades desde el nacimiento, o crear terapias para resolver problemas del lenguaje y aprender más de éste como un sistema de comunicación innato de nuestra especie.

Aun cuando la distancia que hay entre cada nivel de la memoria humana es abismal (genética, lingüística y cognitiva), la influencia recíproca entre estos es evidente, pues mientras lo genético trabaja a nivel molecular y se expresa en los rasgos biológicos de nuestra especie, equipada con un cerebro de aproximadamente 100 mil millones de neuronas; lo lingüístico trabaja a nivel mental, materializándose en las distintas lenguas de la humanidad que recrean el mundo en cada palabra; y en el plano de los conocimientos se expresa en palabras y actividades grupales que atesoran el conocimiento de pueblos que por siglos han sabido mantener el entorno en el que viven y lo preservan para sus descendientes y para el resto del mundo.

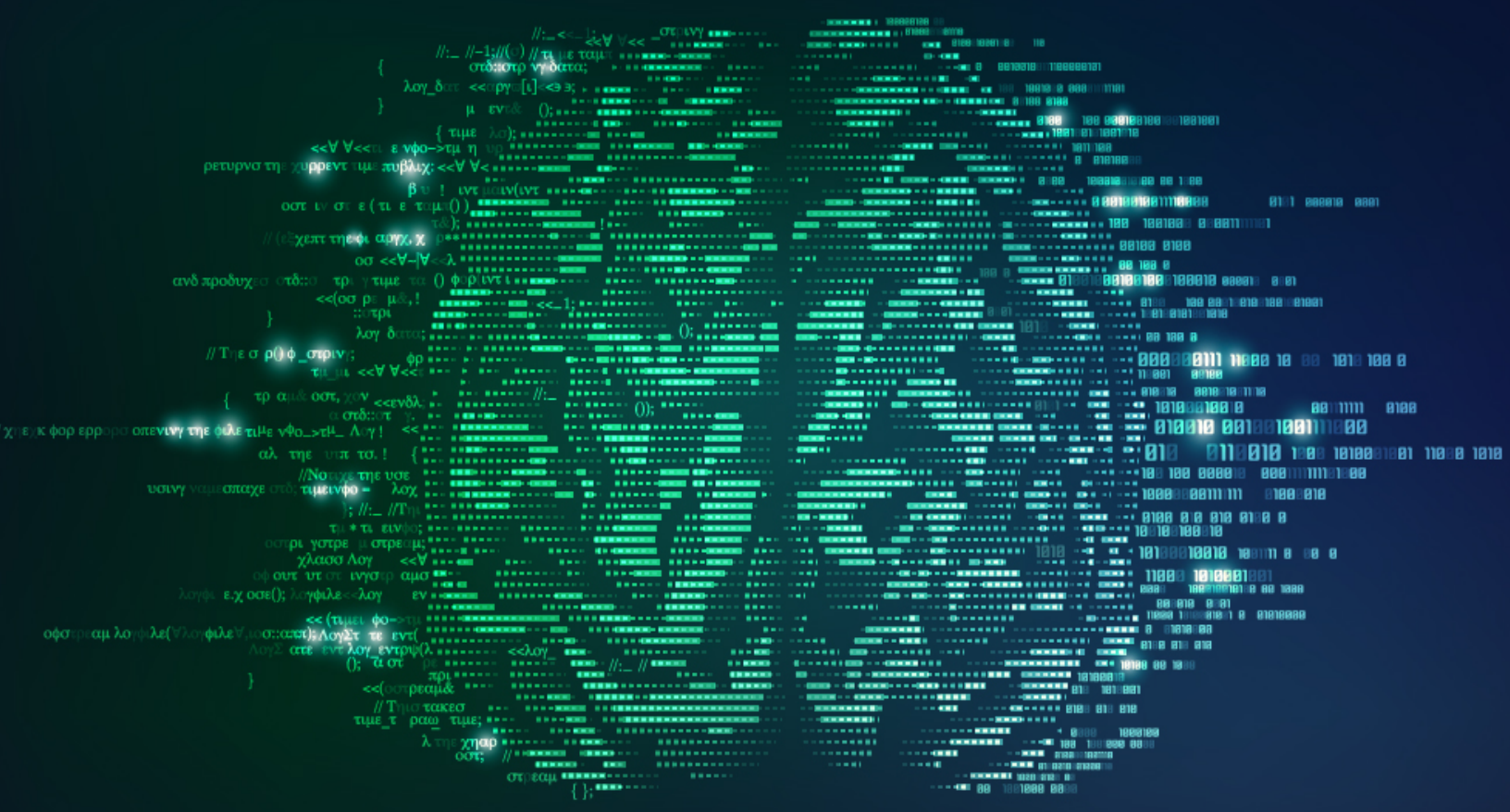




\section{La memoria, expresión de la vida}

El cuerpo humano es consecuencia de la diversidad que ha evolucionado por millones de años para alcanzar un orden tan complejo que apenas comenzamos a entender en algunos de sus procesos, donde orden y diversidad han crecido uno a la par del otro; Víctor Toledo afirma que "desde una perspectiva termodinámica, el orden, que es la complejidad que existe en el universo, aumenta proporcionalmente con la diversidad [...] Por esta razón, la evolución cósmica postula que la variedad aumenta a medida que aumenta el orden" (2008: 16).

De esta forma el trabajo colectivo que ejercen las células que forman los tejidos de cada órgano en todo ser vivo se remonta a las primeras épocas de la vida, cuando los organismos unicelulares aprendieron que la supervivencia dependía de su capacidad para colaborar en tareas específicas para perpetuarse, dicha estrategia se propagó a los siguientes niveles de la vida.

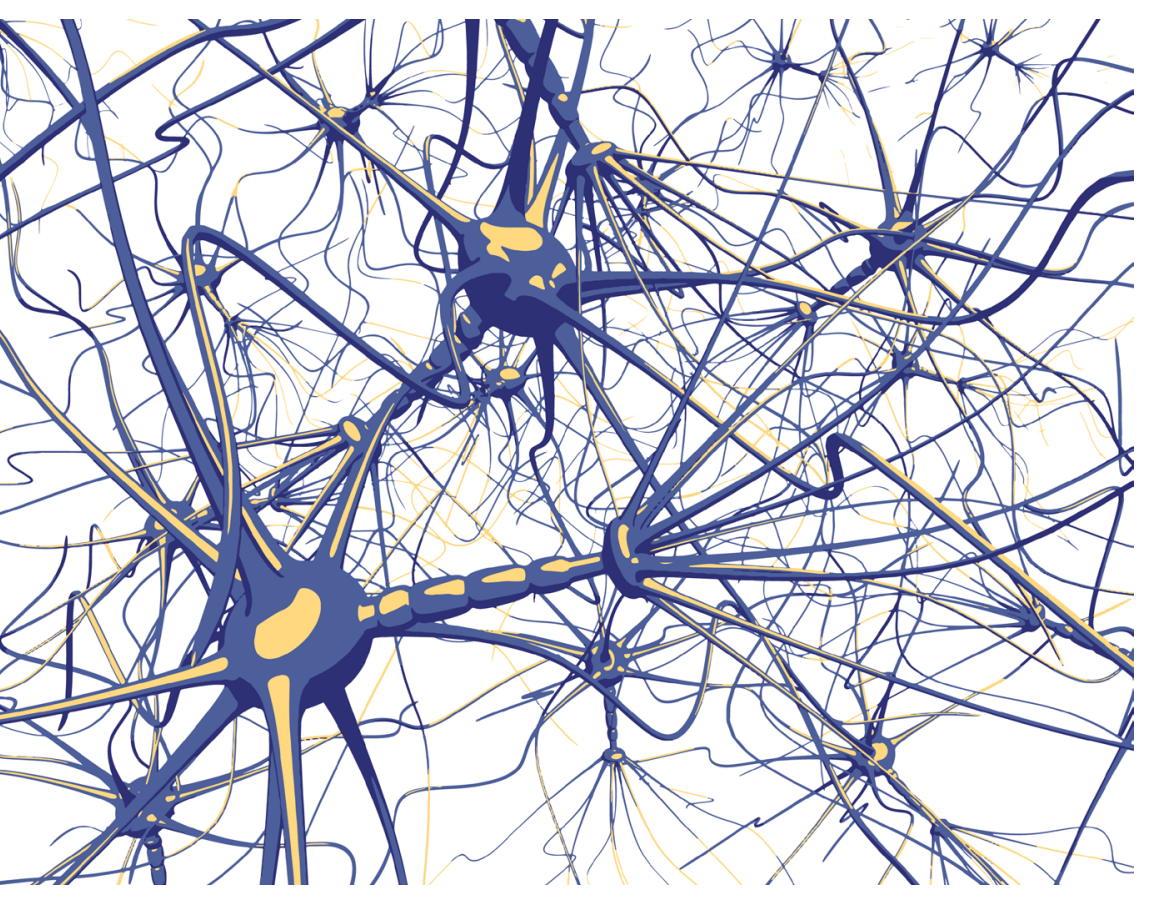
hasta los órganos más sofisticados como el cerebro humano, la cooperación ha sido la base de la supervivencia y la evolución de los seres vivos, perpetuando estos modelos cooperativos a nivel de las células de los individuos, de las especies y de los sistemas ecológicos, donde la comunicación tiene una función de vital importancia, pues la vida necesita transmitir energía, materia e información en estos niveles para continuar como proyecto de diversidad y orden.

La cooperación entre las células cerebrales para evocar recuerdos requiere de conexiones al azar, en lo que se conoce como plasticidad cerebral; ésta es la respuesta al refuerzo de una actividad o experiencia que se ha 
tenido. La memoria y el aprendizaje se generan a partir de las conexiones que se producen en las terminaciones neuronales llamadas dendritas, así como de la creación de éstas, las cuales activan grupos numerosos de neuronas para esta actividad, lo que representa un cambio estructural en el cerebro durante la formación de la memoria (Balderas y Bermúdez, 2007), por lo que la actividad de las neuronas no sólo representa un flujo continuo de energía eléctrica, sino la transformación constante del tejido cerebral a partir de la for-

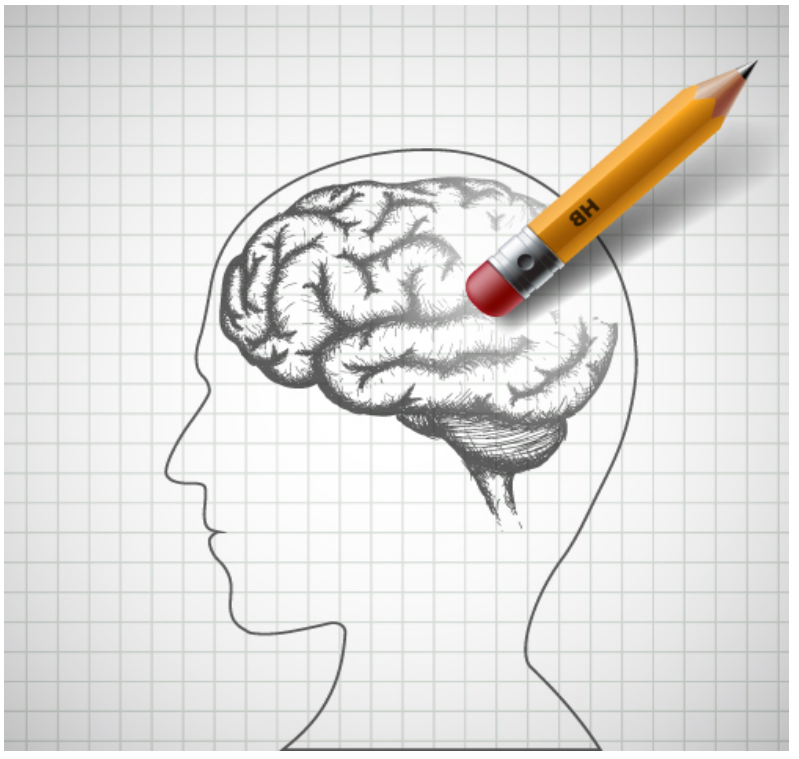
mación de la memoria.

Pero la incorporación de los procesos cooperativos del entorno en el cerebro representa algo más que adaptarse a los cambios del exterior; también adopta las formas de organización autónoma del entorno que permiten su recuperación a partir de sucesos perturbadores o destructores del mismo. Se ha demostrado que los ecosistemas poseen la capacidad de regenerarse y cicatrizar su espacio a partir de microorganismos y especies propias del suelo y, por lo mismo, la salud del suelo en un ecosistema opera como la base de su memoria ecológica (Montenegro, 2004). Lo anterior permite recuperar las especies nativas, así como las relaciones vitales entre éstas, además de reconstruir la dinámica de un ecosistema.

Esto nos permitiría hablar de una semejanza entre la organización del entorno natural con la organización del cerebro a nivel neuronal y equilibrar las relaciones entre sus componentes. Los flujos de materia y energía que los mantienen, posibilitan la recuperación de las perturbaciones que los propios sistemas propician, o de algunas externas que no atenten contra la base de su vida.

\section{Sólo el recuerdo queda}

A pesar de su alto grado de organización y diversidad, la vida tiende al agotamiento y a la desaparición, todo ser vivo muere; los cuerpos interactúan en el espacio y crean la dimensión temporal, además del desgaste y la transformación continua que los hará esfumarse. La frase que Marx escribiera en su 
Manifiesto Comunista encierra un grado de nostalgia y orfandad cuando expresa que "todo lo sólido se desvanece en el aire" para referirse a la pérdida de valor que tiene la vida cuando nos quedamos solos frente a nuestras condiciones reales de existencia en términos sociales; en este nivel también trabaja la cooperación entre los sujetos, para reconstruir algo tan escurridizo y necesario como la memoria.

Como ya se dijo, echar a andar la memoria significa poner a trabajar grandes grupos de seres vivos que, sin importar la escala (células, individuos, especies o ecosistemas), se relacionan entre sí para reconstruir la información ante una necesidad o ambiente que requiere de tal ejercicio. La recuperación del pasado es una forma de afirmar el presente y proyectarse en el futuro; por lo tanto, recordar en colectivo es una actividad social y política de los sujetos para ejercitar de manera abierta su palabra y acciones.

Los sujetos, como productos y productores de la sociedad, crean rasgos colectivos que les permiten diferenciarse de otros al tiempo que crean recuerdos en común y los atesoran en la tradición oral o en registros de todo tipo, pues la memoria de un colectivo tiende a corromperse y los recuerdos se modifican o se pierden, ya que "en el primer plano de la memoria de un grupo se descomponen los recuerdos de los acontecimientos y experiencias que se refieren a la mayoría de sus miembros, y que resultan de la propia vida..." (Hallbwachs, 2004: 45). Aun cuando estos recuerdos no garantizan la veracidad de lo ocurrido, son clave en la construcción de la identidad para estos grupos porque les permite negociar con la realidad, resaltando, modificando o eliminando hechos del pasado.

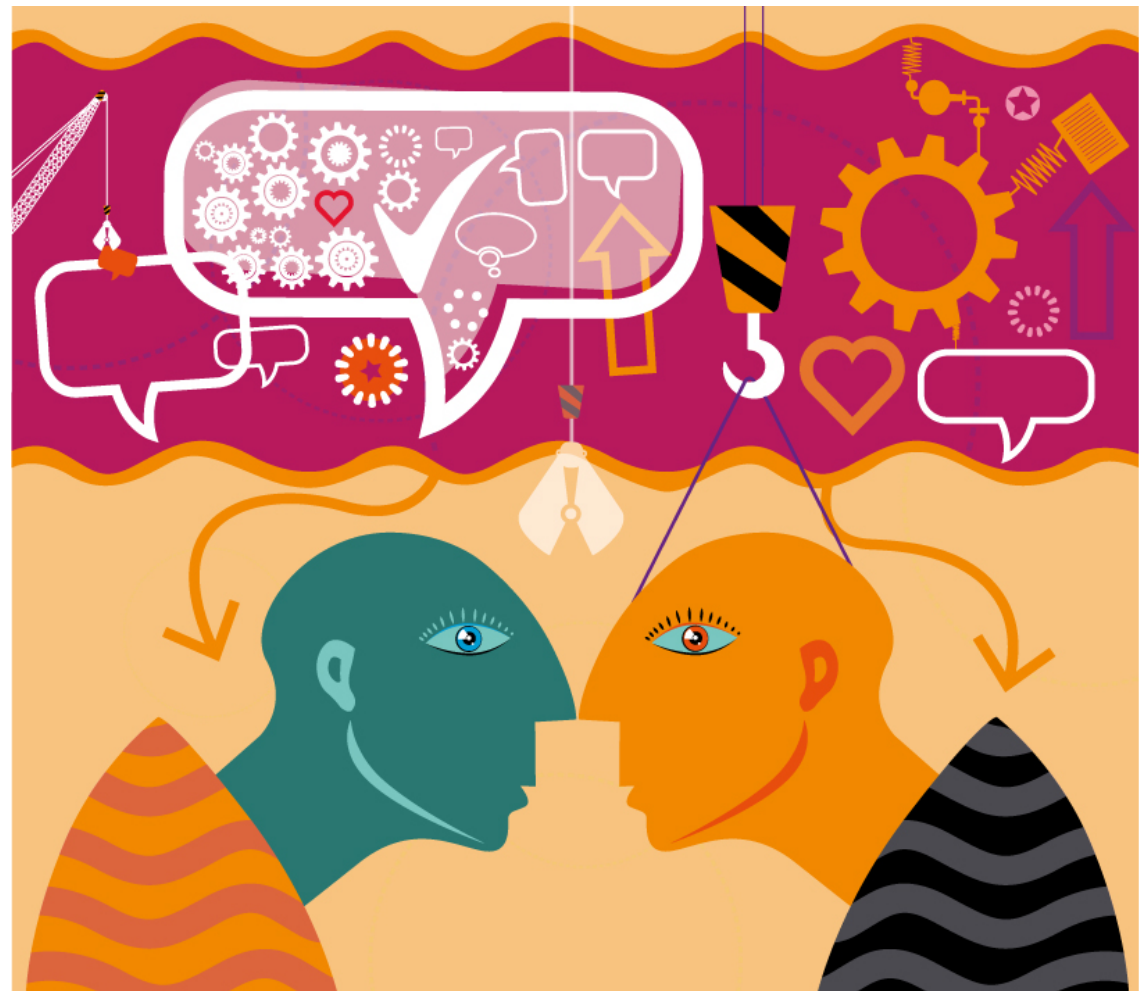


Además de su faceta relacionada con el conocimiento, la memoria tiene otra parte que es emotiva, pues no hay evocación que no se vea impregnada de emoción, ya que la palabra recuerdo, en su raíz latina significa re (volver hacia atrás) cordis (corazón), volver a algo mediante el corazón; y esto nos hace ver que la emoción es parte fundamental de la memoria, pues ahora sabemos que la capacidad de retener sucesos, lugares o personas en la mente implican la emoción. No importa la naturaleza de las emociones, la mente las utiliza como ancla de la memoria. De tal forma que hay recuerdos placenteros que se evocan una y otra vez, y otros que por su violencia podrán ser eliminados de la memoria de un grupo de manera inconsciente o selectiva, pero siempre con la finalidad de conservar la identidad y estabilidad del grupo social.

Existe otra forma de olvido social que ocurre cuando se manipulan o destruyen las bases materiales en las cuales se deposita la memoria de un grupo, "olvido que puede encumbrarse omitiendo, relegando o destruyendo memorias. Memorias que pueden desdibujarse demoliendo los materiales con que se crean." (Mendoza, 20015: 130), lo que de inmediato nos lleva a pensar en la destrucción de templos y códices por parte de los españoles al momento de su llegada, por lo que recordar para muchos grupos es una forma de resistencia frente al invasor y al poderoso que busca borrar la memoria de los oprimidos.

A partir del amarre emocional y de la dimensión política entendemos que la memoria no sólo se encuentra funcionando en lo microscópico y de manera silenciosa, ejecutando en cada célula de nuestro cuerpo el programa genético que heredó de nuestros antepasados; también nace de lo individual como una muestra de la memoria colectiva que se forma con la participación de todos los que vivieron en el pasado y que viven en el presente de una comunidad y que utilizan el lenguaje como un marco general para transmitirla y reconstruirla; y se manifiesta más allá de los grupos humanos, en las relaciones que establecen comunidades de especies nativas de un ecosistema para reconstruir el entorno en el que viven cuando su hábitat ha sido perturbado.

\section{Conclusiones}

La complejidad y la transdisciplina todavía tienen mucho que aportar al mundo contemporáneo, sobre todo ante la necesidad de enfrentar problemas que nacen de las condiciones sociales, económicas, políticas, culturales y ecológicas actuales, que exigen formas novedosas e incluyentes para abordar los problemas. El tema de la memoria es un ejemplo de cómo se pueden tender puentes hacia terrenos poco visibles desde un pensamiento lineal y mecánico. Vemos cómo un nivel puede influir en otro, desde la genética hasta el conocimiento, la memoria aparece como hilo conductor de la especie humana. 
También se ha observado cómo la memoria es resultado de una estrategia más amplia que han desarrollado los seres vivos en su evolución para sobrevivir exitosamente. La memoria no sólo es un mecanismo de conocimiento que trabaja en lo individual, sino un patrimonio colectivo que alcanza su mayor punto cuando los sujetos comparten esos conocimientos y refuerzan el saber grupal, fortaleciendo a la sociedad a la que pertenecen. La colaboración permite que sobreviva el más apto debido a que coopera con su comunidad y aporta trabajo, saberes, recuerdos, imágenes y formas de convivencia entre sus semejantes.

La fortaleza del grupo se expresa muchas veces en situaciones de riesgo, cuando se enfrenta condiciones o grupos adversos que atentan contra su integridad, y la memoria colectiva recupera sucesos, lugares, personajes que activan el sentido de origen, los cuales se cubren de símbolos para mover a la emoción e impulsan otros tipos de memoria, siendo la emoción un componente básico de la memoria que involucra lo genético, al liberar hormonas que preparan al cuerpo para situaciones críticas o favorables; lo lingüístico, en las lenguas que atesoran una forma de recrear el mundo; y lo cognitivo al representar conocimientos que han sido transmitidos por generaciones en un entorno cada vez más hostil para las culturas ancestrales frente a los riesgos que representa el mudo actual para muchas sociedades que resisten una globalización que violenta su forma de ser, pues en última instancia la memoria es una forma de conservar la identidad y la vida en sociedad.

\section{Referencias}

* Balderas, M. I. y Bermúdez, F. (2007). Modificaciones estructurales del cerebro durante la formación de la memoria, en Ojeda Martínez, Rosa y Mercadillo Caballero, Roberto, De las neuronas a la cultura. Ensayos multidisciplinarios sobre cognición, (pp. 103-114). México, INAH-ENAH.

* Consuegra, N. (2010). Diccionario de psicología, Bogotá, Ecoe Ediciones.

* Halbwachs, M. (2004). La memoria colectiva. Zaragoza, Prensas Universitarias de Zaragoza.

* Hoebeke, J. (2014). Breve historia del concepto de la cooperación en la evolución. Recuperado de http://www.jaimelago.org

* Mendoza García, J. (2015). Sobre memoria colectiva. Marcos sociales, artefactos e historia, México, UPN.

* Montenegro, R., et. al. (2004). Biología evolutiva humana, Córdoba, Editorial Brujas.

- Morin, E. (1986). El Método. El conocimiento del conocimiento, Madrid, Cátedra.

* Morin, E. (2001). Introducción al pensamiento complejo. Gedisa, Barcelona.

* Morin, E. (2002). El método. La vida de la vida. Madrid, Cátedra. 
Nicolescu, B. (1996). La Transdisciplinariedad. Manifiesto. Mónaco: Ediciones Du Rocher.

* Toledo, V. y Bassols, N. (2008). Memoria biocultural. La importancia ecológica de las sabidurías tradicionales. Barcelona, Icaria Editorial.

\section{Cómo CITAR ESTe ARTículo}

* Ochoa Flores, Oscar (2018). La memoria en tiempos de la complejidad. Revista Digital Universitaria (RDU). Vol. 19, núm. 2 marzo-abril. DOI: http://doi.org/10.22201/ codeic.16076079e.2018.v19n1.a6. 